\title{
Attractive Coulomb interaction, temperature-dependent hybridization, and natural circular dichroism in $1 T$-TiSe 2
}

\author{
C. W. Chuang $\odot,{ }^{1}$ Y. Tanaka, ${ }^{2}$ M. Oura, ${ }^{2}$ K. Rossnagel $\odot,{ }^{3,4}$ and A. Chainani $\oplus^{1,2}$ \\ ${ }^{1}$ National Synchrotron Radiation Research Center, Hsinchu 30076, Taiwan \\ ${ }^{2}$ RIKEN SPring-8 Center, 1-1-1 Kouto, Sayo-cho, Hyogo 679-5148, Japan \\ ${ }^{3}$ Institut für Experimentelle und Angewandte Physik, Christian-Albrechts-Universität zu Kiel, 24098 Kiel, Germany \\ ${ }^{4}$ Ruprecht-Haensel-Labor, Deutsches Elektronen-Synchrotron DESY, 22607 Hamburg, Germany
}

(Received 5 May 2020; revised 6 October 2020; accepted 7 October 2020; published 2 November 2020)

\begin{abstract}
We study the electronic structure of single crystal $1 T$ - $\mathrm{TiSe}_{2}$ using temperature $(T)$-dependent soft $\mathrm{x}$-ray photoemission spectroscopy (PES) and natural circular dichroism (NCD) across the charge-density-wave (CDW) transition at $T_{\mathrm{cdw}} \sim 200 \mathrm{~K}$. We investigate detailed changes in energy positions and widths of the Ti $2 p$ and Se $3 d$ core-level spectra across $T_{\text {cdw }}$. The Ti $2 p$ - $3 d$-resonant PES shows a clear Ti $3 d$ character two-hole correlation satellite in the valence band. A Cini-Sawatzky analysis indicates an effective attractive two-hole Coulomb interaction energy $U_{d d}=-1.3 \mathrm{eV}$. This attractive Coulomb energy is significantly larger than the known energy scale of excitonic correlations $(<100 \mathrm{meV})$ in $1 T$-TiSe ${ }_{2}$. On-resonant $T$-dependent measurements indicate that the strongly hybridized Ti $3 d$-Se $4 p$ bands show increased hybridization in the CDW phase. On-resonant valence band spectra show a finite, possibly experimental geometry-related NCD of the states at and near the Fermi level which do not show $T$ dependence across $T_{\text {cdw }}$. Given the presence of excitonic effects and the periodic lattice distortion, our results suggest that theoretical models need to take into account the attractive Coulomb interaction and hybridization changes for a complete understanding of the CDW in $1 T-\mathrm{TiSe}_{2}$.
\end{abstract}

DOI: 10.1103/PhysRevB.102.195102

\section{INTRODUCTION}

Quasi-two-dimensional bulk, multilayer, and monolayer transition metal dichalcogenides (TMDs) show many special properties and continue to attract enormous attention. Interesting phenomena in the $2 \mathrm{H}$-type TMDs include a charge-density-wave (CDW) transition in $2 \mathrm{H}-\mathrm{TaSe}_{2}$ [1], the co-existence of CDW order and superconductivity in $2 \mathrm{H}$ $\mathrm{NbSe}_{2}[2,3]$, a superconducting dome in the band insulator $2 \mathrm{H}-\mathrm{MoS}_{2}$ obtained by liquid and solid gating [4,5], and so on. The $1 T$-type TMDs also show CDW order in $1 T-\mathrm{TaS}_{2}$, $1 T-\mathrm{TaSe}_{2}, 1 T-\mathrm{TiSe}_{2}$, and so on [1,6], coexistence of CDW and superconductivity in $\mathrm{Cu}$-intercalated $1 T-\mathrm{TiSe}_{2}$ [7], and pressure-induced superconductivity with CDW order in $1 T$ $\mathrm{TaS}_{2}$ [8], and $1 T-\mathrm{TiSe}_{2}$ [9]. The monolayer systems show fascinating properties which are due to correlation effects and not seen in the bulk, such as nonhydrogenic Rydberg states in $\mathrm{WS}_{2}$ [10], evidence for Ising pairing in superconducting $\mathrm{NbSe}_{2}$ in the presence of a CDW [11], and spin-polarized electrons in $\mathrm{MoS}_{2}$ [12].

In this work, we study the prototypical CDW material $1 T-\mathrm{TiSe}_{2}$ which has been the focus of several important experimental and theoretical investigations. The CDW transition at $T_{\text {cdw }} \sim 200 \mathrm{~K}$ was first reported nearly 45 years ago by Di Salvo et al. and the authors speculated that the $2 \times 2 \times 2$ CDW transition was driven by an electron-hole coupling, i.e., it had an excitonic origin [6]. The role of electron-hole coupling was also concluded from an angleresolved photoemission spectroscopy (ARPES) study, which identified a Se $4 p$ hole band lying just below the Fermi level $\left(\mathrm{E}_{F}\right)$ at the $\Gamma$-point and a $\mathrm{Ti} 3 d$ character electron band crossing the $\mathrm{E}_{F}$ at the $L$-point, but along with an indirect Jahn-Teller (J-T) effect [13]. On the other hand, Rossnagel et al. analyzed their ARPES results in conjunction with a simple two-band model and concluded that their photoemission results were consistent with electrical transport and Hall coefficient measurements, and favored a band J-T effect [14]. An early synchrotron x-ray thermal diffuse scattering study had identified the softening of a $L_{1}$ phonon [15], consistent with a microscopic theory suggesting a band J-T effect driving the transition [16]. Subsequent high-resolution inelastic x-ray scattering studies confirmed a complete softening of a transverse optical phonon at the $L$-point suggesting a conventional electron-phonon coupling mechanism for the structural instability and CDW transition in $1 T-\mathrm{TiSe}_{2}$ [17]. However, ARPES studies reported by Cercellier et al. were interpreted using a Bardeen-Cooper-Schrieffer (BCS)-like model for calculating the spectra of the normal phase and an excitonic insulator type CDW phase [18]. The authors concluded that the CDW transition was induced by an exciton condensation. This was based on model calculations which reproduced the backfolding of the $\Gamma$-centered $\operatorname{Se} 4 p$ hole bands and a very weak backfolding of the $L$-point centered $\mathrm{Ti} 3 d_{z^{2}}$ character electron band at $E_{F}$.

Very recently, quantum oscillation and magnetotransport measurements showed a Fermi surface (FS) reconstruction, with a small electron FS dominating the electronic properties at low temperature while an electron and a hole FS exist at $T>T_{\text {cdw }}$ [19]. Momentum-resolved electron energy loss 
spectroscopy studies reported on collective electronic modes and also concluded an exciton condensation driven CDW, in which the phonons only dress up the excitons and create a periodic lattice distortion (PLD) [20]. On the other hand, a time-resolved spectroscopy study was used for simultaneously probing the ultrafast terahertz response of PLD-related phonons and electronic conductivity, and it showed that the PLD persists even when a femtosecond infrared pulse selectively melts the excitonic order [21]. The results showed that the PLD and excitonic orders are coupled in $1 T-\mathrm{TiSe}_{2}$, consistent with theoretical results which invoked a cooperative $\mathrm{J}-\mathrm{T}$ effect and excitonic order [22]. In fact, in a very recent theoretical study using real-time time-dependent density functional calculations, it was concluded that the excitonic order and PLD mutually amplify each other, resulting in the CDW transition [23].

On the theoretical front, in general, the band dispersions and Fermi surfaces of most of the TMDs in bulk form can be explained by band structure calculations in the absence of on-site Coulomb correlations. However, there are a few TMDs where experimental features are not reproduced correctly in local density approximation (LDA) calculations without on-site correlations. For example, in $1 T-\mathrm{HfTe}_{2}$, an experimentally observed electron band at the $M$ point in the Brillouin zone could not be correctly reproduced by LDA calculations [24]. As discussed above, the monolayer TMDs do show evidence for Coulomb interactions [10-12]. For $1 T$ $\mathrm{TiSe}_{2}$, it was theoretically shown that the CDW instability is obtained in the absence of on-site Coulomb correlations [25]. However, including the on-site repulsive Coulomb correlations suppressed the CDW instability. On the other hand, the authors could show that a nonlocal exchange interaction was important to obtain the CDW state via formation of a $p$ - $d$ hybridized state leading to an enhanced electron-phonon coupling [25]. Thus, it is important to experimentally investigate the presence or absence of correlation effects as well as the role of hybridization in the electronic structure of $1 T-\mathrm{TiSe}_{2}$. Further, it has been reported that $1 T-\mathrm{TiSe}_{2}$ exhibits a chiral CDW order [26], but this is still under debate [27]. Very recently, a study showed gyrotropic order in $1 T-\mathrm{TiSe}_{2}$, in the form of chiral order induced by midinfrared circularly polarized light in an originally achiral lattice [28]. Hence, we also carried out a check for NCD as a signature of chirality in $1 T-\mathrm{TiSe}_{2}$.

We report a study of the Se $3 d$ and Ti $2 p$ core levels, Ti $2 p-3 d \mathrm{XAS}$, and resonant valence band PES of single crystal $1 T-\mathrm{TiSe}_{2}$ above and below $T_{\mathrm{cdw}}$. We observe small changes in the Se $3 d$ and $\mathrm{Ti} 2 p$ core level spectra which reflect the changes reported for $\mathrm{Se} 4 p$ and $\mathrm{Ti} 3 d$ valence band states. XAS measurements were carried out with clockwise (CW) and counterclockwise (CCW) circularly polarized $\mathrm{x}$-rays to check for NCD in the unoccupied conduction band states. Ti $2 p-3 d$ resonant PES was measured to check for NCD in the valence band states. Resonant PES identifies a Ti $3 d$ character two-hole correlation satellite which is analyzed using the Cini-Sawatzky method [29-32]. It provides an effective attractive Coulomb interaction energy $U_{d d}=-1.3 \mathrm{eV}$ between two valence holes. This attractive energy is significantly larger than the attractive excitonic correlation energy scale $(<100 \mathrm{meV})$ in $1 T-\mathrm{TiSe}_{2}$ [20,21]. Our results suggest that the attractive on-site Coulomb interaction and $T$-dependent hybridization are important and theoretical models need to take this into account for a complete understanding of the CDW in $1 T-\mathrm{TiSe}_{2}$.

\section{EXPERIMENTAL METHODS}

The samples used in this study are bulk single crystals of $1 T-\mathrm{TiSe}_{2}$ grown by iodine chemical vapor transport [33]. A mixture of high-purity $\mathrm{Ti}$ and Se with a slight Se excess was placed in an evacuated quartz ampoule together with iodine as a transport agent. The ampoule was heated in a four zone furnace under a temperature gradient of $665-555^{\circ} \mathrm{C}$ for $600 \mathrm{~h}$. The method yielded $1 T-\mathrm{TiSe}_{2}$ crystals of typically $3 \times 3 \times 0.15 \mathrm{~mm}^{3}$. Electrical resistivity measurements confirmed a CDW transition temperature $T_{\text {cdw }} \sim 200 \mathrm{~K}$ [34].

Soft $x$-ray core-level PES, XAS, and resonant PES experiments were carried out at beamline BL17SU, SPring-8, Japan. The XAS measurements (total electron yield mode) and the PES experiments were performed using a spectrometer equipped with a VG-Scienta 2002 analyzer [35]. The NCD measurements were done using $\mathrm{CW}$ and $\mathrm{CCW}$ circularly polarized $\mathrm{x}$-rays as seen looking along the incident beam direction. The $\mathrm{x}$-ray beam has a nominal spot size of $\sim 40$ micron diameter. The experimental geometry was normal incidence into the $a b$ plane for the XAS and resonant PES measurements. The resonant PES spectra were collected in angle-integrated mode. The total energy resolution for the Ti $2 p-3 d$ resonant PES and the core-level photoemission spectra (measured with $h v=700 \mathrm{eV}$ ) was set to $200 \mathrm{meV}$ and the accuracy of determining $E_{F}$ was $\pm 10 \mathrm{meV}$. The energy reference $E_{F}$ and energy resolutions for the soft x-ray core level and resonant PES measurements were determined from the Fermi edge of a gold film at $T=32 \mathrm{~K}$. All results reported here were obtained from samples cleaved in an ultra-high vacuum $\left(1 \times 10^{-9}\right.$ mbar $)$ preparation chamber with a scotch tape and immediately transferred into the main chamber at $1 \times 10^{-10}$ mbar. The measurements were carried out at $T=$ 32 and $300 \mathrm{~K}$ and the samples were cooled using a liquid-He flow-type cryostat.

\section{RESULTS AND DISCUSSION}

Figure 1 shows the Se $3 d$ and Ti $2 p$ core-level PES spectra of $1 T-\mathrm{TiSe}_{2}$ measured with an incident photon energy $h v=$ $700 \mathrm{eV}$ above and below $T_{\mathrm{cdw}}$, at $T=300$ and $32 \mathrm{~K}$. As seen in Fig. 1(a), the Se $3 d$ spectra show single peaks for the Se $3 d_{5 / 2}$ and Se $3 d_{3 / 2}$ spin-orbit split features. The spectrum at $T=32 \mathrm{~K}$ is shifted to a higher binding energy (BE) compared to the $T=300 \mathrm{~K}$ spectrum. Similarly, Fig. 1(d) shows the Ti $2 p$ spectra consisting of the Ti $2 p_{3 / 2}$ and Ti $2 p_{1 / 2}$ spin-orbit split features, and here also the $T=32 \mathrm{~K}$ spectrum is shifted to a higher BE compared to the $T=300 \mathrm{~K}$ spectrum. The Se $3 d$ peak shapes indicate that both the $3 d_{5 / 2}$ and $3 d_{3 / 2}$ peaks show a narrowing at $T=32 \mathrm{~K}$. From a least-squares fitting of the spectra using asymmetric Doniach-Sunjic (DS) lineshapes at $T=300 \mathrm{~K}$ [Fig. 1(b)], the Se $3 d_{5 / 2}$ and Se $3 d_{3 / 2}$ features are positioned at a BE of 53.29 and $54.13 \mathrm{eV}$, respectively. For $T=32 \mathrm{~K}$ [Fig. 1(c)], the peaks are positioned at 53.35 and $54.20 \mathrm{eV}$, indicating a shift of about 60 to $70 \mathrm{meV}$. For the $\mathrm{Ti}$ 

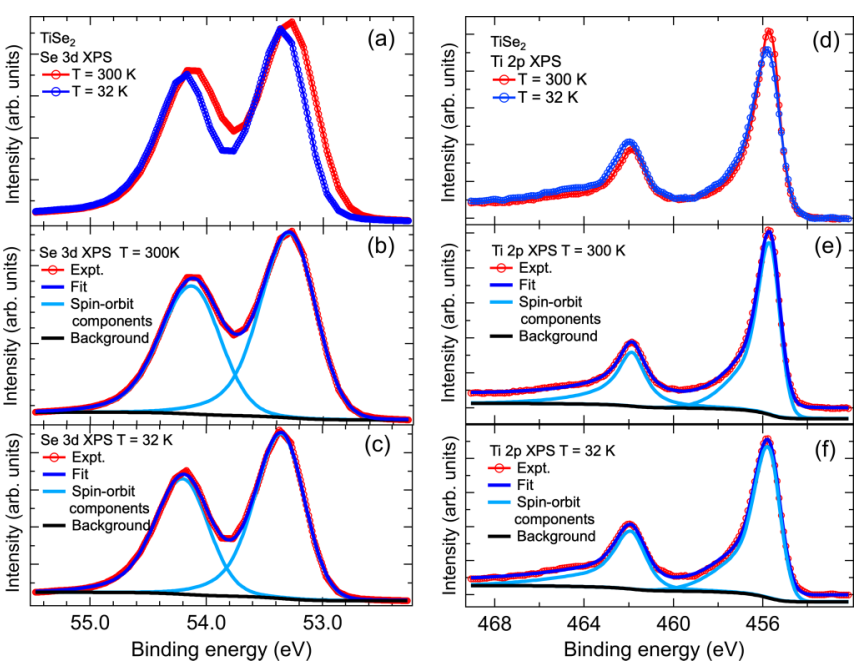

FIG. 1. (a)-(c) Experimental Se $3 d$ core level PES spectra of $\mathrm{TiSe}_{2}$ at $T=32$ and $300 \mathrm{~K}$. The data show a clear shift to higher binding energy (BE) and narrowing for the $T=32 \mathrm{~K}$ spectrum compared to the $T=300 \mathrm{~K}$ spectrum, across the CDW transition at $T_{\text {cdw }}=200 \mathrm{~K}$. (d)-(f) Experimental Ti $2 p$ core level PES spectra of $\mathrm{TiSe}_{2}$ at $T=32$ and $300 \mathrm{~K}$. The data show small changes in $\mathrm{BE}$ and spectral shapes for the $T=32 \mathrm{~K}$ spectrum compared to the $T=300 \mathrm{~K}$ spectrum.

$2 p$ core level PES spectra, a careful look at the peak shapes indicate that both the $2 p_{3 / 2}$ and $2 p_{1 / 2}$ peaks show a small broadening at $T=32 \mathrm{~K}$ compared to $T=300 \mathrm{~K}$. A similar fitting of the Ti $2 p$ spectra using DS lineshapes at $T=300 \mathrm{~K}$ [Fig. 1(e)] shows that the Ti $2 p_{3 / 2}$ and Ti $2 p_{1 / 2}$ features are positioned at a BE of 455.71 and $461.87 \mathrm{eV}$, respectively. For $T=32 \mathrm{~K}$ [Fig. 1(f)], the corresponding peaks are positioned at 455.78 and $461.93 \mathrm{eV}$, indicating again a shift of about 60 to $70 \mathrm{meV}$. The obtained fitting parameters for $\mathrm{Se} 3 d$ and $\mathrm{Ti}$ $2 p$ levels are listed in Tables I and II.

Given an error bar $\pm 10 \mathrm{meV}$ in the accuracy of the BEs, a shift of 60 to $70 \mathrm{meV}$ in both the Se $3 d$ and Ti $2 p$ spectra is consistent with the shift of the Ti $3 d_{z^{2}}$ conduction band minimum: It was shown that the $\mathrm{Ti} 3 d_{z^{2}}$ conduction band minimum shifts from just above $E_{F}$ to $\sim 60 \mathrm{meV}$ below $E_{F}$ at the $L$-point (along the $A-L$ direction), as observed in ARPES studies of bulk $1 T$-TiSe 2 across the CDW transition $[13,14,18,21,36,37]$. Since the shift of the Ti $3 d_{z^{2}}$ band corresponds to an increase of the chemical potential, we

TABLE I. Fitting parameters for the Se $3 d$ core level spectra discussed in Figs. 1(a) to 1(c)

\begin{tabular}{lccc}
\hline \hline Se $3 d$ & $\begin{array}{c}\text { Binding energy } \\
(\mathrm{eV})\end{array}$ & $\begin{array}{c}\text { FWHM } \\
(\mathrm{eV})\end{array}$ & $\begin{array}{c}\text { Asymmetry } \\
\text { parameter }\end{array}$ \\
$T=32 \mathrm{~K}$ & & 0.51 & 0.22 \\
$3 d_{5 / 2}$ & 53.35 & 0.55 & 0.15 \\
$3 d_{3 / 2}$ & 54.20 & & \\
$T=300 \mathrm{~K}$ & & 0.56 & 0.18 \\
$3 d_{5 / 2}$ & 53.29 & 0.61 & 0.16 \\
$3 d_{3 / 2}$ & 54.13 & & \\
\hline \hline
\end{tabular}

TABLE II. Fitting parameters for the Ti $2 p$ core level spectra discussed in Figs. 1(d) to 1(f).

\begin{tabular}{lccc}
\hline \hline Ti $2 p$ & $\begin{array}{c}\text { Binding energy } \\
(\mathrm{eV})\end{array}$ & $\begin{array}{c}\text { FWHM } \\
(\mathrm{eV})\end{array}$ & $\begin{array}{c}\text { Asymmetry } \\
\text { parameter }\end{array}$ \\
$T=32 \mathrm{~K}$ & & & \\
\hline $2 p_{3 / 2}$ & 455.78 & 1.50 & 0.35 \\
$2 p_{1 / 2}$ & 461.93 & 1.79 & 0.24 \\
$T=300 \mathrm{~K}$ & & & \\
$2 p_{3 / 2}$ & 455.71 & 1.28 & 0.35 \\
$2 p_{1 / 2}$ & 461.87 & 1.59 & 0.15 \\
\hline \hline
\end{tabular}

attribute the observed core-level shifts in the Se $3 d$ and Ti $2 p$ spectra to a global chemical potential shift.

It is noted that the high symmetry momentum points $\Gamma$, $M, A$, and $L$ in the Brillouin zone of the high-temperature normal phase all become equivalent (this point is labeled $\left.\Gamma^{*}\right)$ due to band folding associated with the low-temperature $2 \times 2 \times 2$ CDW phase [33,38]. Thus, although the shift of the Se $4 p_{x, y}$ valence band edge at the $\Gamma$-point was reported to be slightly higher $(\sim 100 \mathrm{meV})[33,38]$ and our in-plane angle integrated measurements with $h v=700 \mathrm{eV}$ have a $k_{z}$ value corresponding to the $\Gamma$ point of the high-temperature normal phase, the core level shifts in the CDW phase are due to the global chemical potential shift and independent of the Se $4 p_{x, y}$ band shift associated with the $\Gamma$-point. A smaller shift of the Se $3 d$ core levels between 280 and $70 \mathrm{~K}(\sim 25 \mathrm{meV})$ [38] and a reduction of the gap by $\approx 60 \mathrm{meV}$ at the $\Gamma^{*}$-point associated with the $A$-point derived top of the Se $4 p_{x, y}$ band edge and the bottom of the Ti $3 d_{z^{2}}$ band was reported recently using higher resolution ARPES [33]. It is noted that the core level changes are unlike changes seen for the CDW transition in $1 T-\mathrm{TaS}_{2}[39,40]$ which showed a splitting of Ta $4 f$ peaks in the low- $T$ CDW phase. The splitting was attributed to charge transfer between distinct sites $[39,40]$ and its absence in $1 T-\mathrm{TiSe}_{2}$ suggests negligible charge transfer between distinct sites in the CDW phase. The same shift in core level PES for the Se $3 d$ and Ti $2 p$ core levels also indicates that the shifts are not due to charge transfer from Se $4 p$ to Ti $3 d$-derived states.

In Fig. 2, we plot the Ti $L$-edge $(2 p-3 d)$ XAS spectra at $T=32$ and $T=300 \mathrm{~K}$, obtained with $\mathrm{CW}$ and $\mathrm{CCW}$ circularly polarized x-rays. The spectra are similar to the ones reported in earlier studies [41-43]. The peaks at $455.7 \mathrm{eV}$ ( "c") and $457.5 \mathrm{eV}$ (三 "e") for the $L_{3}$ edge (and at $461.4 \mathrm{eV}$ ( " "h") and $463.2 \mathrm{eV}$ (三 "j") for the $L_{2}$ edge) originate from the $t_{2 g}$ and $e_{g}$ crystal field split states, respectively. The CW and CCW spectra at $T=32 \mathrm{~K}$ and at $T=300 \mathrm{~K}$ show no obvious polarization dependent changes. This result indicates negligible NCD in the XAS spectra of the CDW phase and the non-CDW phase. On the other hand, a small but finite $T$-dependent change between the two phases is observed in the $\mathrm{CW}$ and $\mathrm{CCW}$ profiles. The inset shows the leading edge of the $L_{3}$-edge main peak on an expanded scale. The leading edge of the $L_{3}$-edge main peak shifts to higher photon energy by $\sim 80 \mathrm{meV}$ at half maximum of the peak intensity for the $T=32 \mathrm{~K}$ spectrum compared to the $T=300 \mathrm{~K}$ spectrum. This is very close to the 60 to $70 \mathrm{meV} \mathrm{BE}$ shift observed in the 


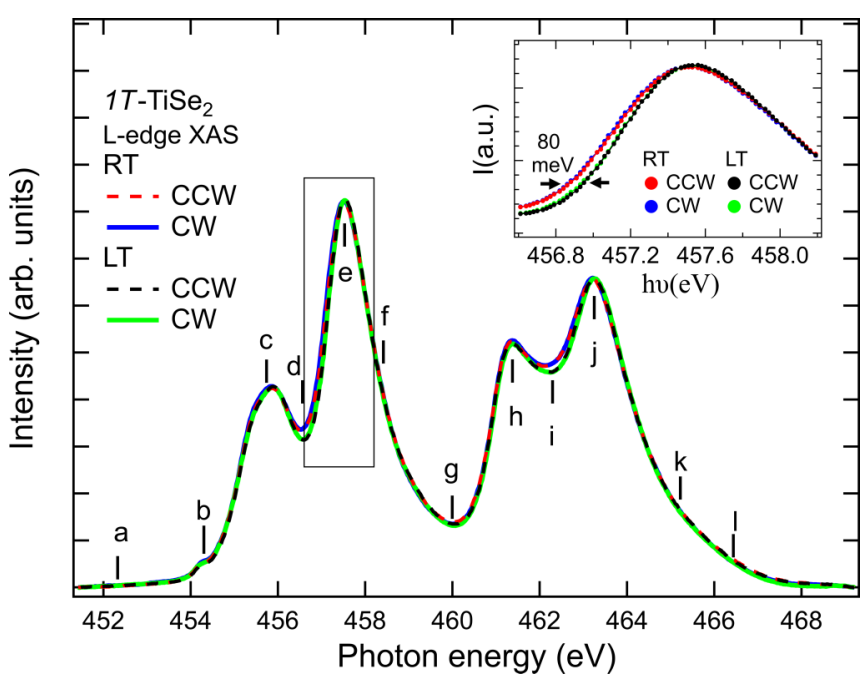

FIG. 2. Experimental Ti $2 p-3 d$ XAS spectra of $\mathrm{TiSe}_{2}$ at $T=$ $300 \mathrm{~K}(\mathrm{RT})$ and $T=32 \mathrm{~K}$ (LT) obtained with CW and CCW circularly polarized $\mathrm{x}$-rays. The inset shows the box region of the main panel on an expanded photon energy scale. A shift of $\sim 80 \mathrm{meV}$ in the leading edge of the $L_{3}$-edge main peak is observed between the $T=300 \mathrm{~K}$ and $T=32 \mathrm{~K}$ spectra, but negligible polarizationdependent changes are seen between $\mathrm{CW}$ and $\mathrm{CCW}$ spectra at either temperature. Labels "a"-"l" mark the photon energies used to measure Ti $2 p-3 d$ resonant PES spectra shown in Fig. 3.

Ti $2 p$ core level spectra shown in Fig. 1(d) and indicates that the small changes in XAS spectra are associated with initial state shifts.

Based on a cluster model analysis of XAS spectra [41], it was shown that covalency in $\mathrm{TiX}_{2}(\mathrm{X}=\mathrm{S}, \mathrm{Se}, \mathrm{Te})$ increases on going from $\mathrm{TiS}_{2}$ to $\mathrm{TiTe}_{2}$. Further, band-structure calculations for $\mathrm{TiSe}_{2}$ concluded significant Ti $3 d_{x z, y z}$ and $3 d_{x^{2}-y^{2}, x y}$ character states in the occupied DOS at $\sim 3 \mathrm{eV}$ BE $[44,45]$. This is attributed to $\mathrm{Ti} 3 d$-Se $4 p$ hybridization, although $\mathrm{Ti}$ formally has a $d^{0}$ configuration in $\mathrm{TiSe}_{2}$. To check for Ti $3 d$-Se $4 p$ hybridization effects in $\mathrm{TiSe}_{2}$, we carried out $\mathrm{Ti}$ $2 p$ - $3 d$ resonant PES at $T=32$ and $300 \mathrm{~K}$. The resonant PES spectra measured at photon energies labeled "a" to " $\mathrm{g}$ " $(h v=452.34$ to $460.02 \mathrm{eV}$ ) in the XAS spectrum (Fig. 2) across the $L_{3}$-edge at $T=300 \mathrm{~K}$ are shown in Fig. 3(a). The spectra are normalized for BEs above $15 \mathrm{eV}$ where there are no spectral features. The corresponding $L_{2}$-edge-resonant PES spectra measured using photon energies labeled "g" to "l" ( $h v=460.02$ to $466.34 \mathrm{eV})$ are shown in Fig. 3(b). The energy values of the incident photon energies "a" to "l" are shown in Figs. 3(a) and 3(b). The spectrum measured with photon energy "a" just below the Ti $L_{3}$-edge is the off-resonant valence band spectrum. It consists of three features which originate in Se $4 p$ orbitals, and are spread between $E_{F}$ and about 6-eV BE. The shallow core level at 13.1-eV BE is the $\mathrm{Se}$ $4 s$ feature. As we increase the photon energy from "a" to "e", a systematic increase in the intensity of the feature at $\sim 3 \mathrm{eV}$ $\mathrm{BE}$ is observed with a maximum for incident photon energy "e", and then its intensity gets reduced for energies " $\mathrm{f}$ " and "g". Since photon energy "e" corresponds to the maximum absorption intensity in the XAS spectrum, it indicates that the intensity changes in the valence band arise from Ti $3 d$
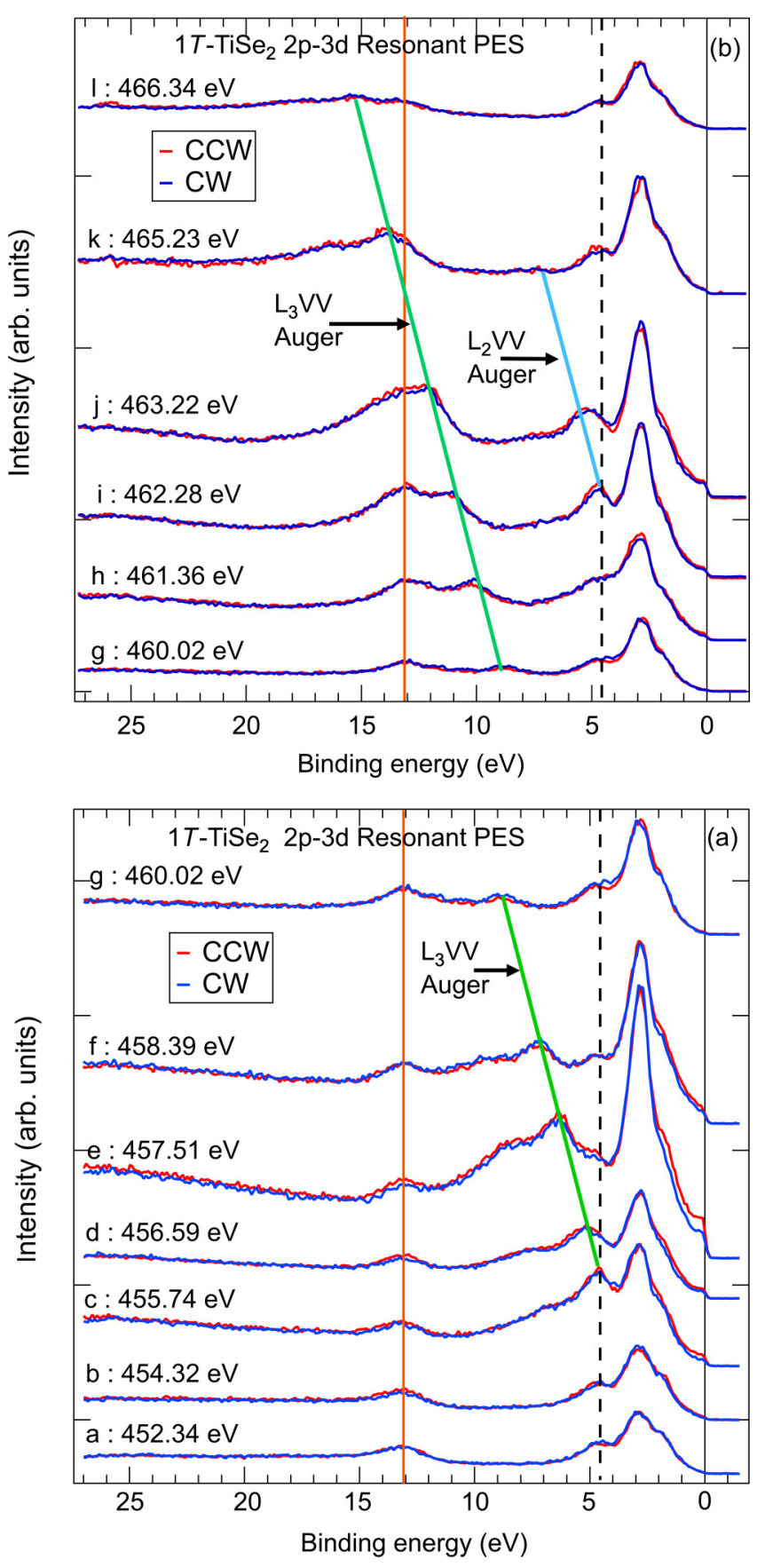

FIG. 3. (a) Experimental Ti $2 p-3 d$ resonant PES spectra of $\mathrm{TiSe}_{2}$ at $T=300 \mathrm{~K}$ at photon energies across the Ti $L_{3}$ edge (labeled "a""g" in Fig. 2) with CW and CCW circular polarization, shifted along the intensity axis for clarity. The data show a resonance maximum at $\sim 3 \mathrm{eV}$ binding energy for spectrum "e". For spectra "c" to "g", a clear $L_{3} V V$ Auger two-hole correlation satellite is marked with a green line. (b) The $2 p-3 d\left(L_{3,2}\right.$-edge) resonant PES data for photon energies labeled "h" to "l" in Fig. 2. The green line shows the systematic shift of the $L_{3} V V$ Auger two-hole correlation satellite for higher photon energies. The $L_{2} V V$ correlation satellite (light blue line) is observed as a weak enhancement and broadening at $\sim 5 \mathrm{eV}$ $\mathrm{BE}$ for the spectrum " $\mathrm{j}$ ”. At higher photon energies, the $L_{2} V V$ correlation satellite is suppressed due to the opening of the Coster-Kronig $L_{2} L_{3} V$ channel at and above the $L_{2}$ threshold. The orange vertical line corresponds to the Se $4 s$ core level and the black dashed line marks a weak DOS feature overlapping the Auger satellite. 
character states hybridized with Se $4 p$ states. This confirms band-structure calculation results which indicate significant Ti $3 d_{x z, y z}$ and $3 d_{x^{2}-y^{2}, x y}$ character states hybridized with Se $4 p$ states at $\sim 3 \mathrm{eV}$ BE. Further, another feature at $\sim 5 \mathrm{eV} \mathrm{BE}$ and a weak broad feature at $\sim 6.5$ to $7.0 \mathrm{eV}$ BE starts increasing in intensity at photon energy "c". The $5-\mathrm{eV}$ feature shifts to higher BEs as the photon energy is increased and tracks the changes in the incident photon energy. This behavior indicates that the feature at $\sim 5 \mathrm{eV} \mathrm{BE}$ in the "c" spectrum is a regular Auger feature and corresponds to the Ti $L_{3} V V$ two-hole correlation satellite in the valence band. Qualitatively similar resonance behavior was reported by Shkvarin et al. [46], but the resonance enhancement was weaker and the correlation satellite was not analyzed to estimate the on-site Coulomb correlation energy.

Figure 3(b) shows the $2 p-3 d$ ( $L_{2}$-edge) resonant PES data for photon energies labeled "g" to "l" in Fig. 2. The systematic shift of the $L_{3} V V$ Auger two-hole correlation satellite continues for the spectra labeled "g" (satellite $\sim 9 \mathrm{eV} \mathrm{BE}$ ) to "l" (satellite at $\sim 15.5 \mathrm{eV} \mathrm{BE}$ ) in Fig. 3(b) (green line). At photon energies "i", "j", and " $\mathrm{k}$ " the two-hole correlation satellite crosses the Se $4 s$ shallow core level and continues to higher binding energies for spectrum "l". At photon energies "i", "j". and "k", the $\sim 3-\mathrm{eV}$ feature again undergoes a resonant enhancement with a maximum for photon energy " $\mathrm{j}$ ", but the $L_{2} V V$ correlation satellite (blue line) shows up only as a weak enhancement and broadening at $\sim 5 \mathrm{eV} \mathrm{BE}$ for the spectrum " $\mathrm{j}$ ". At higher energies for spectrum " $\mathrm{k}$ " and "l" the feature is suppressed. The suppression of the $L_{2} V V$ correlation satellite is due to the opening of the Coster-Kronig $L_{2} L_{3} V$ channel at and above the $L_{2}$ threshold. Following Hüfner et al. [47] we checked the onset of the so-called regular Auger behavior by plotting the kinetic energies of the $L_{3} V V$ and $L_{2} V V$ features versus the photon energy relative to the maximum of the $L_{3}$ and $L_{2}$ peaks. The regular Auger peak onset is estimated to be 1.7 and $0.8 \mathrm{eV}$ below the main peaks of the XAS $L_{3}$ and $L_{2}$ edges, respectively.

Using the Cini-Sawatzky method [29-32] we analyzed the two-hole correlation satellite energy to obtain an experimental measure of on-site element specific Coulomb correlation energy $U_{d d}$. The Cini-Sawatzky method has been reliably used to estimate on-site element-specific Coulomb correlation energies in several $3 d$ transition metal ( $\mathrm{Ti}$ to $\mathrm{Cu}$ ) compounds [48-52]. The method is based on the fact that the Auger transition for a core hole in the initial state and two valence holes in the final state is sensitive to the on-site Coulomb energy if the two holes reside on the same site. To estimate the on-site Coulomb correlation energy, one needs to obtain the two-hole spectrum without correlations. This can be obtained from a numerical self-convolution of the one-hole spectrum obtained from photoemission of the valence band states. The on-site Coulomb energy $\mathrm{U}_{d d}$ obtained from the two hole Auger satellite corresponds to the energy cost for the "reaction" $2 M^{+} \rightarrow M+M^{2+}$, where $M$ is an atom in a solid. Thus, the value of the on-site Coulomb correlation energy between two valence holes, as deduced from Auger spectra, is the difference between the first ionization energy $\left(I_{1}\right)$ and second ionization energy $\left(I_{2}\right)$, i.e., $U_{d d \text { (Auger) }}=I_{2}-I_{1}$. This is expected to be slightly different from the normal definition of the Hubbard on-site Coulomb energy $U_{d d \text { (Hubbard), }}$

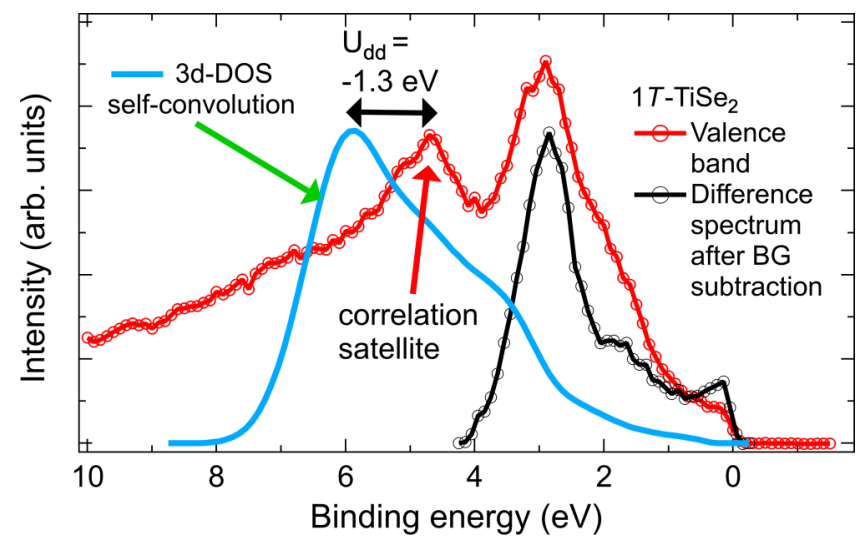

FIG. 4. The self-convoluted Ti $3 d$-partial density of states (blue) compared to the correlation satellite observed in the valence band (red) spectrum "c" of Fig. 3 for estimating $U_{d d}$. The Ti $3 d$ partial DOS is obtained as a difference spectrum (on-resonance spectrum "e" - off resonant spectrum "a") and followed by subtracting a linear background (BG).

which corresponds to the "reaction" $2 M \rightarrow M^{-}+M^{+}$and $U_{d d \text { (Hubbard) }}=I_{1}-A$, i.e., it is the difference between the first ionization energy $I_{1}$ and the electron affinity $A$. It is noted that both the values correspond to the difference in energy between one less electron and one more electron compared to a reference state. However, the reference states are $M$ and $M^{+}$, which are obviously not equal, but the difference in the estimated values of $U_{d d \text { (Auger) }}$ and $U_{d d \text { (Hubbard) }}$ is expected to be small due to screening effects in a solid [53].

For the present case, we use the Ti $L_{3} V V$ Auger satellite to estimate the on-site Coulomb energy due to two Ti character valence holes in the final state. In Fig. 4, we plot the difference spectrum between the off-resonant spectrum "a" [shown in Fig. 3(a)] and on-resonant spectrum "e" [also shown in Fig. 3(a)] to identify and separate out the Ti $3 d$ character partial density of states (i.e., the Ti $3 d$ character one-hole spectrum). Since the feature at 5-eV BE corresponds to the two-hole satellite, we curtail the Ti $3 d$ partial DOS range up to a $\mathrm{BE}$ of $4 \mathrm{eV}$ to obtain the one-hole spectrum and a linear background was subtracted from the spectrum (black line in Fig. 4). A numerical self-convolution of the obtained Ti $3 d$ partial DOS, which represents the two-hole spectrum without correlations, is then compared to the two-hole correlation satellite in spectrum "c". The energy separation between the two-hole correlation satellite peak and the peak of the two-hole spectrum without correlations provides a measure of the average on-site Coulomb energy $U_{d d}$ in the Ti $3 d$ states and we obtain $U_{d d}=-1.3 \pm 0.2 \mathrm{eV}$. Thus we obtain an attractive (negative) on-site Coulomb energy in $1 T-\mathrm{TiSe}_{2}$. This result is qualitatively consistent with an early estimate of $U_{d d}$ provided by deBoer, Haas, and Sawatzky [53], who obtained a value of $U_{d d} \sim-2.5 \mathrm{eV}$ based on Ti $L_{3} V V$ spectra measured using a $\mathrm{Mg} K_{\alpha} \mathrm{x}$-ray source $(h v=1253.6 \mathrm{eV})$. It is noted that our numerical two-hole spectrum also shows a very similar lineshape and energy spread as reported by deBoer $e t$ $a l$. , but due to improved signal to noise ratio in the present data, we obtain a lower value of $U_{d d}=-1.3 \pm 0.2 \mathrm{eV}$. These authors attributed the negative $U_{d d}$ to bipolaron formation: 

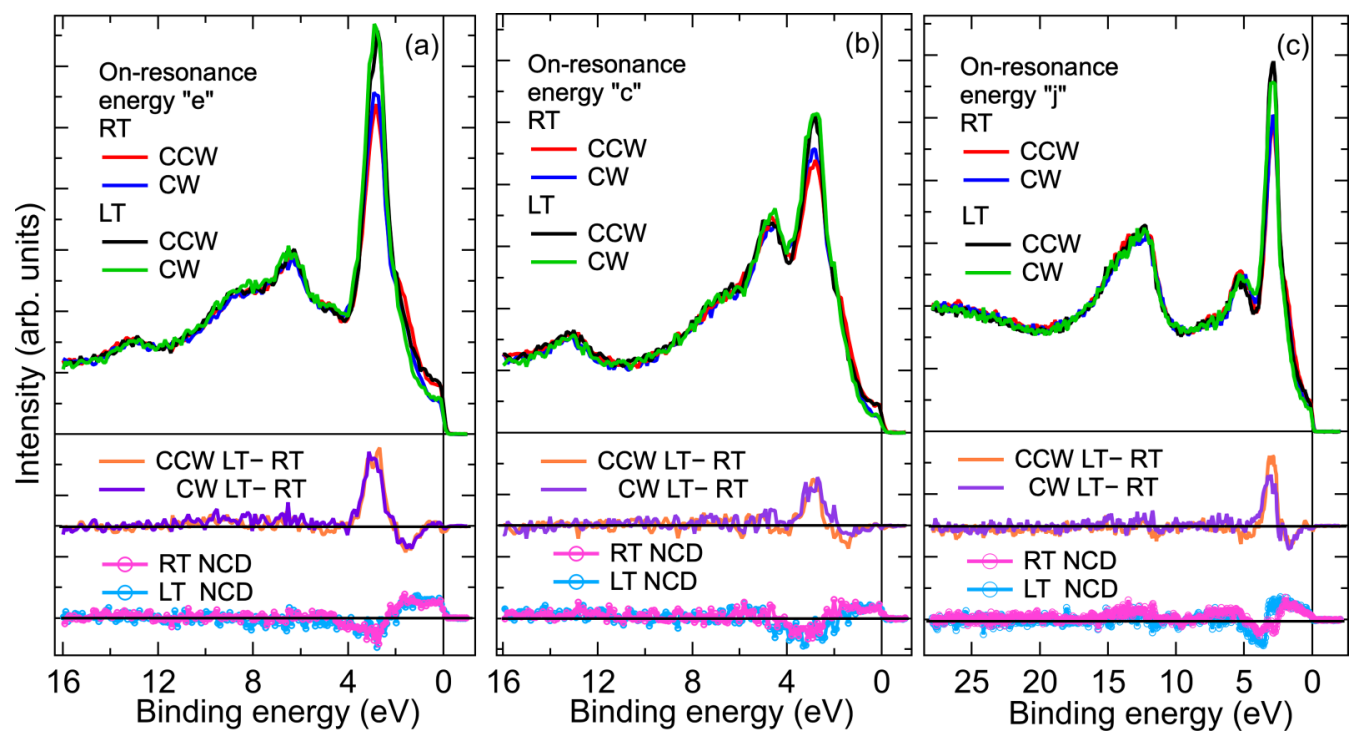

FIG. 5. The circular polarization dependence at $T=32 \mathrm{~K}(\mathrm{LT})$ and $T=300 \mathrm{~K}$ (RT) of the valence band spectrum obtained using photon energies corresponding to: (a) "on-resonance" $\mathrm{e}_{g}$ maximum intensity of the $L_{3}$ XAS labeled "e" (hv $=457.5 \mathrm{eV}$ ), (b) "on-resonance" $t_{2 g}$ maximum intensity of the $L_{3}$ XAS labeled "c" ( $\left.\mathrm{h} v=455.7 \mathrm{eV}\right)$ and (c) "on-resonance" $\mathrm{e}_{g}$ maximum intensity of the $L_{2}$ XAS labeled "j" (hv $=463.2 \mathrm{eV})$. The obtained natural circular dichroism $(\mathrm{NCD}=\mathrm{CCW}-\mathrm{CW})$ at RT and LT is also shown along with the $T$-dependent changes (LT-RT) for CW and CCW polarization in the lower panel.

If the first valence hole creates a potential well in which the second valence hole gets trapped, and the resulting relaxation energy is larger than the repulsive energy between the holes, it would effectively result in a negative $U_{d d}$. The bipolaron formation can arise from a lattice polarization, such as a J-T distortion $[54,55]$.

An effective attractive interaction between electrons (negative- $U$ Hubbard model) can be derived from strong electron-phonon coupling [55-57] or due to a purely electronic origin $[58,59]$. These models were discussed for the superconducting series $\mathrm{Ba}_{1-x} \mathrm{~K}_{x} \mathrm{BiO}_{3}$ and $\mathrm{BaBi}_{1-x} \mathrm{~Pb}_{x} \mathrm{O}_{3}$, which are derived from a three-dimensional CDW parent material, $\mathrm{BaBiO}_{3}$. Since the magnitude of the estimated attractive $U_{d d}=-1.3 \mathrm{eV}$ for $1 T-\mathrm{TiSe}_{2}$ is significantly larger than the attractive excitonic correlations associated with the measured normal-state band gap and characteristic electronic mode energy, which are both smaller than $100 \mathrm{meV}$ in $1 T$ $\mathrm{TiSe}_{2}[20,21,33]$, it may be expected to play an important role in determining the ground state of $1 T-\mathrm{TiSe}_{2}$.

The $T$ - and polarization-dependent spectra over a wide BE scale were measured at photon energies "c", "e", "h", and " $\mathrm{j}$ " corresponding to the peaks due to $\mathrm{t}_{2 g}$ and $\mathrm{e}_{g}$ crystal field split states (Fig. 2). We first discuss the $T$ - and polarization-dependent spectra obtained at photon energy "e" corresponding to the "on-resonance" $e_{g}$ maximum intensity of the $L_{3}$ XAS, as shown in Fig. 5(a). The high intensity feature at $\sim 3 \mathrm{eV}$ and the weaker features between $2 \mathrm{eV} \mathrm{BE}$ and $E_{F}$ show $T$ - and polarization-dependent changes. As discussed above, the $\sim 3 \mathrm{eV}$ feature consists of $\mathrm{Ti} 3 d_{x y, x^{2}-y^{2}}$ and $\mathrm{Ti}$ $3 d_{x z, y z}$ states strongly hybridized with $\mathrm{Se} 4 p$ states, while states from 0 to $2 \mathrm{eV}$ BE have very weak contribution from $\mathrm{Ti} 3 d$ states $[44,45]$. It is noted that the Ti $3 d_{z^{2}}$ character electron band within $50 \mathrm{meV}$ of $E_{F}$ seen in high-resolution ARPES studies $[13,14,18,21,36-38]$ at the $L$-point in the
Brillouin zone is not observed in our angle-integrated data, as our experimental resolution is $200 \mathrm{meV}$. The $\sim 3 \mathrm{eV} \mathrm{BE}$ feature gets clearly enhanced at $T=32 \mathrm{~K}$ compared to $T=$ $300 \mathrm{~K}$ as shown in Fig. 5(a). The increase in spectral weight is not conserved and hence cannot be attributed to charge transfer from the Se $4 p$ states to the Ti $3 d_{x y, x^{2}-y^{2}}$ and Ti $3 d_{x z, y z}$ states in the low- $T$ CDW phase, and this is also consistent with the core level analysis. This indicates that the Ti $3 d$-Se $4 p$ hybridization responsible for the $\sim 3 \mathrm{eV}$ feature becomes stronger in the low- $T$ CDW phase. This is consistent with the decrease in Ti-Se bond distances in the CDW phase, as is known from structural studies [38,60]. The $T$-dependent changes for the $\mathrm{CW}$ and $\mathrm{CCW}$ spectra are plotted as difference curves in the same figure. We also checked for NCD $=\mathrm{CCW}-\mathrm{CW}$, on resonance at $T=32$ and $T=300 \mathrm{~K}$. The NCD spectra are also plotted in the same figure. A clear NCD is seen for the states between 0 to $2 \mathrm{eV} \mathrm{BE}$ followed by a weak dip at $\sim 3 \mathrm{eV}$ BE. Surprisingly, the NCD spectra do not show any $T$-dependent changes across the CDW transition. This suggests that the electronic states do not display a chiral character which can be associated with the CDW transition.

Figures 5(b) and 5(c) show the spectra for photon energies "c" $\left(t_{2 g}\right.$ peak of $L_{3}$ XAS $)$ and "j" ( $e_{g}$ peak of $L_{2}$ XAS $)$, respectively. Qualitatively similar spectra were obtained for photon energy "h" ( $t_{2 g}$ peak of $L_{2}$ XAS, not shown). At these energies also, the high-intensity feature at $\sim 3 \mathrm{eV}$ gets enhanced at $T=32 \mathrm{~K}$ compared to $T=300 \mathrm{~K}$, as seen in Figs. 5(b) and 5(c). The results corroborate the increase in hybridization between the Ti $3 d$ and $\mathrm{Se} 4 p$ states in the CDW phase compared to the room temperature non-CDW phase. We also checked for the NCD at $T=32 \mathrm{~K}$ and $T=300 \mathrm{~K}$ and they are also plotted in Figs. 5(b) and 5(c). Although the NCD signal has lower signal-to-noise ratio for BEs between 2 to $5 \mathrm{eV}$, the negligible differences in the NCD spectra at and 
near the Fermi level for the $T=32$ and $T=300 \mathrm{~K}$ confirms the behavior seen in the on-resonance spectra obtained with energy "e". In a very recent study, using resonant and nonresonant x-ray diffraction, it was shown that $1 T-\mathrm{TiSe}_{2}$ does not show structural chirality [61]. Further, since $1 T-\mathrm{TiSe}_{2}$ is nonmagnetic, the NCD signal cannot arise due to magnetization and can only be due to an electronic chirality or an asymmetric experimental geometry [62]. Since the NCD does not change as a function of temperature across the CDW transition, it suggests the absence of electronic chirality. We speculate that the observed NCD arises from an asymmetric experimental geometry such as a misalignment of the in-plane $\Gamma-M$ axis with respect to the analyzer entrance slit. The NCD probably originates from spin-orbit dichroism [62], which would be consistent with the fact that the electronic states within $\sim 2 \mathrm{eV}$ of $E_{F}$ have $\mathrm{Se} 4 p$ character, which exhibit strong spin-orbit coupling ( $\sim 0.4 \mathrm{eV}$ in the atomic state) [63]. It is considered necessary to carry out high-resolution ARPES measurements on resonance for incident $\mathrm{x}$-rays along the $\mathrm{CDW}$ wave vector $(1 / 2,1 / 2,1 / 2)$ for a more rigorous check of the CDW coupled chiral electronic states in $1 T$ - $\mathrm{TiSe}_{2}$, especially because it was recently shown that the material exhibits strongly $k_{z}$ dependent Ti $3 d$-Se $4 p$ hybridization in the CDW phase [33]. We hope future work can address this issue in a more rigorous way.

\section{CONCLUSION}

In conclusion, we carried out $T$-dependent photoemission spectroscopy and NCD studies across the CDW transition in $1 T$-TiSe 2 . Ti $2 p$ and Se $3 d$ core level spectra show consistent changes across $T_{\mathrm{cdw}}$. A Ti $3 d$ character two-hole correlation satellite provides an effective attractive on-site Coulomb interaction energy $U_{d d}=-1.3 \mathrm{eV}$. On-resonant $T$-dependent measurements indicate that the strongly hybridized Ti $3 d-\mathrm{Se}$ $4 p$ bands show increased hybridization in the CDW phase. The results suggest that the attractive Coulomb interaction and hybridization changes cannot be neglected for a complete understanding of the CDW in $1 T-\mathrm{TiSe}_{2}$. It would be interesting to quantify the on-site Coulomb energies in other $3 d$ TMDs where correlations are known to be important [10-12], thereby leading to a better understanding of the CDW formation, superconductivity, and magnetism in these materials.

\section{ACKNOWLEDGMENTS}

A.C. thanks the Ministry of Science and Technology (MOST) of Taiwan, Republic of China, for financially supporting this research under Contract No. MOST 108-2112M-213-001-MY3. The synchrotron radiation experiments were performed at BL17SU of SPring-8 with the approval of RIKEN SPring-8 Center (Proposal No. 20140019).
[1] J. A. Wilson, F. J. Di Salvo, and S. Mahajan, Charge-density waves and superlattices in the metallic layered transition metal dichalcogenides, Adv. Phys. 24, 117 (1975).

[2] T. Yokoya, T. Kiss, A. Chainani, S. Shin, M. Nohara, and H. Takagi, Fermi surface sheet-dependent superconductivity in $2 \mathrm{H}-\mathrm{NbSe}_{2}$, Science 294, 2518 (2001).

[3] T. Kiss, T. Yokoya, A. Chainani, S. Shin, T. Hanaguri, M. Nohara, and H. Takagi, Charge-order-maximized momentumdependent superconductivity, Nat. Phys. 3, 720 (2007).

[4] J. T. Ye, Y. J. Zhang, R. Akashi, M. S. Bahramy, R. Arita, and Y. Iwasa, Superconducting dome in a gate-tuned band insulator, Science 338, 1193 (2012).

[5] Y. Saito, Y. Nakamura, M. S. Bahramy, Y. Kohama, J. T. Ye, Y. Kasahara, Y. Nakagawa, M. Onga, M. Tokunaga, T. Nojima, Y. Yanase, and Y. Iwasa, Superconductivity protected by spinvalley locking in ion-gated $\mathrm{MoS}_{2}$, Nat. Phys. 12, 144 (2016).

[6] F. J. Di Salvo, D. E. Moncton, and J. V. Waszczak, Electronic properties and superlattice formation in the semimetal $\mathrm{TiSe}_{2}$, Phys. Rev. B 14, 4321 (1976).

[7] E. Morosan, H. W. Zandbergen, B. S. Dennis, J. W. G. Bos, Y. Onose, T. Klimczuk, A. P. Ramirez, N. P. Ong, and R. J. Cava, Superconductivity in $\mathrm{Cu}_{x} \mathrm{TiSe}_{2}$, Nat. Phys. 2, 544 (2006).

[8] B. Sipos, A. F. Kusmartseva, A. Akrap, H. Berger, L. Forro, and E. Tutis, From Mott state to superconductivity in $1 T-\mathrm{TaS}_{2}$, Nat. Mater. 7, 960 (2008).

[9] A. F. Kusmartseva, B. Sipos, H. Berger, L. Forro, and E. Tutis, Pressure Induced Superconductivity in Pristine $1 T-\mathrm{TiSe}_{2}$, Phys. Rev. Lett. 103, 236401 (2009).

[10] A. Chernikov, T. C. Berkelbach, H. M. Hill, A. Rigosi, Y. Li, O. B. Aslan, D. R. Reichman, M. S. Hybertsen, and T. F. Heinz, Exciton Binding Energy and Nonhydrogenic Rydberg Series in Monolayer WS $\mathrm{W}_{2}$, Phys. Rev. Lett. 113, 076802 (2014).
[11] X. Xi, Z. Wang, W. Zhao, J.-H. Park, K. T. Law, H. Berger, L. Forro, J. Shan, and K. F. Mak, Evidence of Ising pairing in superconducting $\mathrm{NbSe}_{2}$ atomic layers, Nat. Phys. 12, 139 (2016).

[12] J. G. Roch, G. Froehlicher, N. Leisgang, P. Makk, K. Watanabe, T. Taniguchi, and R. J. Warburton, Spin-polarized electrons in monolayer $\mathrm{MoS}_{2}$, Nat. Nanotechnol. 14, 432 (2019).

[13] T. E. Kidd, T. Miller, M. Y. Chou, and T. C. Chiang, ElectronHole Coupling and the Charge Density Wave Transition in $\mathrm{TiSe}_{2}$, Phys. Rev. Lett. 88, 226402 (2002).

[14] K. Rossnagel, L. Kipp, and M. Skibowski, Charge-densitywave phase transition in 1T-TiSe2: Excitonic insulator versus band-type Jahn-Teller mechanism, Phys. Rev. B 65, 235101 (2002).

[15] M. Holt, P. Zschack, H. Hong, M. Y. Chou, and T.-C. Chiang, X-Ray Studies of Phonon Softening in TiSe 2 , Phys. Rev. Lett. 86, 3799 (2001).

[16] Y. Yoshida and K. Motizuki, Electron lattice interaction and lattice instability of $1 T-\mathrm{TiSe}_{2}$, J. Phys. Soc. Jpn. 49, 898 (1980).

[17] F. Weber, S. Rosenkranz, J.-P. Castellan, R. Osborn, G. Karapetrov, R. Hott, R. Heid, K.-P. Bohnen, and A. Alatas, Electron-Phonon Coupling and the Soft Phonon Mode in $\mathrm{TiSe}_{2}$, Phys. Rev. Lett. 107, 266401 (2011).

[18] H. Cercellier, C. Monney, F. Clerc, C. Battaglia, L. Despont, M. G. Garnier, H. Beck, P. Aebi, L. Patthey, H. Berger, and L. Forro, Evidence for an Excitonic Insulator Phase in $1 T-\mathrm{TiSe}_{2}$, Phys. Rev. Lett. 99, 146403 (2007).

[19] P. Knowles, B. Yang, T. Muramatsu, O. Moulding, J. Buhot, C. J. Sayers, E. Da Como, and S. Friedemann, Fermi Surface Reconstruction and Electron Dynamics at the Charge-DensityWave Transition in $\mathrm{TiSe}_{2}$, Phys. Rev. Lett. 124, 167602 (2020). 
[20] A. Kogar, M. S. Rak, S. Vig, A. A. Husain, F. Flicker, Y. Il Joe, L. Venema, G. J. MacDougall, T. C. Chiang, E. Fradkin, J. van Wezel, and P. Abbamonte, Signatures of exciton condensation in a transition metal dichalcogenide, Science 358, 1314 (2017).

[21] M. Porer, U. Leierseder, J.-M. Menard, H. Dachraoui, L. Mouchliadis, I. E. Perakis, U. Heinzmann, J. Demsar, K. Rossnagel, and R. Huber, Non-thermal separation of electronic and structural orders in a persisting charge density wave, Nat. Mater. 13, 857 (2014).

[22] J. van Wezel, P. Nahai-Williamson, and S. S. Saxena, Excitonphonon interactions and superconductivity bordering charge order in $\mathrm{TiSe}_{2}$, Phys. Rev. B 83, 024502 (2011).

[23] C. Lian, S.-J. Zhang, S.-Q. Hu, M.-X. Guan, and S. Meng, Ultrafast charge ordering by self-amplified exciton-phonon dynamics in $\mathrm{TiSe}_{2}$, Nat. Commun. 11, 43 (2020).

[24] Y. Nakata, K. Sugawara, A. Chainani, K. Yamauchi, K. Nakayama, S. Souma, P.-Y. Chuang, C.-M. Cheng, T. Oguchi, K. Ueno, T. Takahashi, and T. Sato, Dimensionality reduction and band quantization induced by potassium intercalation in 1T-HfTe 2 , Phys. Rev. Materials 3, 071001(R) (2019).

[25] M. Hellgren, J. Baima, R. Bianco, M. Calandra, F. Mauri, and L. Wirtz, Critical Role of the Exchange Interaction for the Electronic Structure and Charge-Density-Wave Formation in $\mathrm{TiSe}_{2}$, Phys. Rev. Lett. 119, 176401 (2017).

[26] J. Ishioka, Y. H. Liu, K. Shimatake, T. Kurosawa, K. Ichimura, Y. Toda, M. Oda, and S. Tanda, Chiral Charge-Density Waves, Phys. Rev. Lett. 105, 176401 (2010).

[27] B. Hildebrand, T. Jaouen, M.-L. Mottas, G. Monney, C. Barreteau, E. Giannini, D. R. Bowler, and P. Aebi, Local RealSpace View of the Achiral $1 T-\mathrm{TiSe}_{2} 2 \times 2 \times 2$ Charge Density Wave, Phys. Rev. Lett. 120, 136404 (2018).

[28] S.-Y. Xu, Q. Ma, Y. Gao, A. Kogar, A. Zong, A. M. Mier Valdivia, T. H. Dinh, S.-M. Huang, B. Singh, C.-H. Hsu, T.-R. Chang, J. P. C. Ruff, K. Watanabe, T. Taniguchi, H. Lin, G. Karapetrov, D. Xiao, P. Jarillo-Herrero, and N. Gedik, Spontaneous gyrotropic electronic order in a transition-metal dichalcogenide, Nature 578, 545 (2020).

[29] M. Cini, Density of states of two interacting holes in a solid, Solid State Commun. 20, 605 (1976)

[30] M. Cini, Two hole resonances in the XVV Auger spectra of solids, Solid State Commun. 24, 681 (1977).

[31] G. A. Sawatzky, Quasiatomic Auger Spectra in Narrow-Band Metals, Phys. Rev. Lett. 39, 504 (1977).

[32] M. Cini, Comment on quasiatomic Auger spectra in narrowband metals, Phys. Rev. B 17, 2788 (1978).

[33] M. D. Watson, O. J. Clark, F. Mazzola, I. Markovi, V. Sunko, T. K. Kim, K. Rossnagel, and P. D. C. King, Orbital- and kzSelective Hybridization of Se $4 p$ and Ti $3 d$ States in the Charge Density Wave Phase of $\mathrm{TiSe}_{2}$, Phys. Rev. Lett. 122, 076404 (2019)

[34] M. D. Watson, A. M. Beales, and P. D. C. King, On the origin of the anomalous peak in the resistivity of $\mathrm{TiSe}_{2}$, Phys. Rev. B 99, 195142 (2019).

[35] K. Horiba, N. Kamakura, K. Yamamoto, K. Kobayashi, and S. Shin, A high-resolution angle-resolved photoemission spectrometer combined with laser molecular-beam epitaxy at SPring-8 BL17SU, J. Electron. Spectrosc. Relat. Phenom. 144147, 1027 (2005).

[36] T. Rohwer, S. Hellmann, M. Wiesenmayer, C. Sohrt, A. Stange, B. Slomski, A. Carr, Y. Liu, L. M. Avila, M. Kallane, S.
Mathias, L. Kipp, K. Rossnagel, and M. Bauer, Collapse of long-range charge order tracked by time-resolved photoemission at high momenta, Nature 471, 490 (2011).

[37] P. Chen, Y.-H. Chan, X.-Y. Fang, S.-K. Mo, Z. Hussain, A.V. Fedorov, M. Y. Chou, and T.-C. Chiang, Hidden order and dimensional crossover of the charge density waves in $\mathrm{TiSe}_{2}$, Sci. Rep. 6, 37910 (2016).

[38] A. Wegner, J. Zhao, J. Li, J. Yang, A. A. Anikin, G. Karapetrov, K. Esfarjani, D. Louca, and U. Chatterjee, Evidence for pseudoJahn-Teller distortions in the charge density wave phase of $1 T$ $\mathrm{TiSe}_{2}$, Phys. Rev. B 101, 195145 (2020).

[39] H. P. Hughes and J. A. Scarfe, Site Specific Photohole Screening in a Charge Density Wave, Phys. Rev. Lett. 74, 3069 (1995).

[40] K. Ishizaka, T. Kiss, T. Yamamoto, Y. Ishida, T. Saitoh, M. Matsunami, R. Eguchi, T. Ohtsuki, A. Kosuge, T. Kanai, M Nohara, H. Takagi, S. Watanabe, and S. Shin, Femtosecond core-level photoemision spectroscopy on $1 \mathrm{~T}-\mathrm{TaS}_{2}$ using a 60 eV laser source, Phys. Rev. B 83, 081104(R) (2011).

[41] A. S. Shkvarin, Yu. M. Yarmoshenko, N. A. Skorikov, M. V. Yablonskikh, A. I. Merentsov, E. G. Shkvarina, and A. N. Titov, Electronic structure of titanium dichalcogenides $\operatorname{TiX}_{2}(\mathrm{X}=\mathrm{S}$ Se, Te), J. Exp. Theor. Phys. 114, 150 (2012).

[42] C. Monney, K. J. Zhou, H. Cercellier, Z. Vydrova, M. G. Garnier, G. Monney, V. N. Strocov, H. Berger, H. Beck, T. Schmitt, and P. Aebi, Mapping of Electron-Hole Excitations in the Charge-Density-Wave System $1 T-\mathrm{TiSe}_{2}$ Using Resonant Inelastic X-Ray Scattering, Phys. Rev. Lett. 109, 047401 (2012).

[43] B. Pal, Y. Cao, X. Liu, F. Wen, M. Kareev, A. T. N'Diaye, P. Shafer, E. Arenholz, and J. Chakhalian, Anomalous orbital structure in two-dimensional titanium dichalcogenides, Sci. Rep. 9, 1896 (2019).

[44] Y. Gu, Y. Katsura, T. Yoshino, H. Takagi, and K. Taniguchi, Rechargeable magnesium-ion battery based on a $\mathrm{TiSe}_{2}$-cathode with d-p orbital hybridized electronic structure, Sci. Rep. 5, 12486 (2015).

[45] A. R. Jurelo, R. A. Pontes Ribeiro, S. R. de Lazaro, and J. F. H. Monteiro, Structural, vibrational and electronic properties of the superconductor $\mathrm{Cu} x \mathrm{TiSe}_{2}$ : theoretical and experimental insights, Phys. Chem. Chem. Phys. 20, 27011 (2018).

[46] A. S. Shkvarin, Yu. M. Yarmoshenko, N. A. Skorikov, M. V. Yablonskikh, A. I. Merentsov, E. G. Shkvarina, and A. N. Titov, Resonance photoelectron spectroscopy of $\mathrm{TiX}_{2}(\mathrm{X}=\mathrm{S}, \mathrm{Se}, \mathrm{Te})$ titanium dichalcogenides, J. Exp. Theor. Phys. 115, 798 (2012).

[47] S. Hufner, S.-H. Yang, B. S. Mun, C. S. Fadley, J. Schafer, E. Rotenberg, and S. D. Kevan, Observation of the two-hole satellite in $\mathrm{Cr}$ and $\mathrm{Fe}$ metal by resonant photoemission at the $2 \mathrm{p}$ absorption energy, Phys. Rev. B 61, 12582 (2000)

[48] D. van der Marel, J. van Elp, G. A. Sawatzky, and D. Heitmann, X-ray photoemission, bremsstrahlung isochromat, Auger-electron, and optical spectroscopy studies of $\mathrm{Y}-\mathrm{Ba}-\mathrm{Cu}-\mathrm{O}$ thin films, Phys. Rev. B 37, 5136 (1988).

[49] A. Balzarotti, M. De Crescenzi, N. Motta, F. Patella, and A. Sgarlata, Valence charge fluctuations in $\mathrm{YBa}_{2} \mathrm{Cu}_{3} \mathrm{O}_{7-\delta}$ from core-level spectroscopies, Phys. Rev. B 38, 6461 (1988).

[50] A. Chainani, M. Mathew, and D. D. Sarma, Electronspectroscopy study of the semiconductor-metal transition in $\mathrm{La}_{1-x} \mathrm{Sr}_{x} \mathrm{CoO}_{3}$, Phys. Rev. B 46, 9976 (1992).

[51] Y. Ishida, R. Eguchi, M. Matsunami, K. Horiba, M. Taguchi, A. Chainani, Y. Senba, H. Ohashi, H. Ohta, and S. Shin, Coherent 
and Incoherent Excitations of Electron-Doped $\mathrm{SrTiO}_{3}$, Phys. Rev. Lett. 100, 056401 (2008).

[52] A. Chainani, M. Sicot, Y. Fagot-Revurat, G. Vasseur, J. Granet, B. Kierren, L. Moreau, M. Oura, A. Yamamoto, Y. Tokura, and D. Malterre, Evidence for Weakly Correlated Oxygen Holes in the Highest- $T c$ Cuprate Superconductor $\mathrm{HgBa}_{2} \mathrm{Ca}_{2} \mathrm{Cu}_{3} \mathrm{O}_{8+\delta}$, Phys. Rev. Lett. 119, 057001 (2017).

[53] D. K. G. deBoer, C. Haas, and G. A. Sawatzky, Auger spectra of compounds of Sc, Ti and Cr, J. Phys. F: Met. Phys. 14, 2769 (1984).

[54] P. W. Anderson, Model for the Electronic Structure of Amorphous Semiconductors, Phys. Rev. Lett. 34, 953 (1975).

[55] T. M. Rice and L. Sneddon, Real-Space and k-Space Electron Pairing in $\mathrm{BaPb}_{1-x} \mathrm{Bi}_{x} \mathrm{O}_{3}$, Phys. Rev. Lett. 47, 689 (1981).

[56] E. Jurczek and T. M. Rice, A charge-density-wave instability caused by strong electron-phonon coupling in $\mathrm{BaBi}_{1-x} \mathrm{~Pb}_{x} \mathrm{O}_{3}$, Europhys. Lett. 1, 225 (1986).

[57] D. Yoshioka and H. Fukuyama, A simple model for superconductor-semiconductor transition, J. Phys. Soc. Jpn. 54, 2996 (1985).
[58] C. M. Varma, Missing Valence States, Diamagnetic Insulators, and Superconductors, Phys. Rev. Lett. 61, 2713 (1988).

[59] A. Taraphder, H. R. Krishnamurthy, Rahul Pandit, and T. V. Ramakrishnan, Negative-U extended Hubbard model for doped barium bismuthates, Phys. Rev. B 52, 1368 (1995).

[60] S. Kitou, A. Nakano, S. Kobayashi, K. Sugawara, N. Katayama, N. Maejima, A. Machida, T. Watanuki, K. Ichimura, S. Tanda, T. Nakamura, and H. Sawa, Effect of $\mathrm{Cu}$ intercalation and pressure on excitonic interaction in $1 T$-TiSe 2 , Phys. Rev. B 99, 104109 (2019).

[61] H. Ueda, M. Porer, J. R. L. Mardegan, S. Parchenko, N. Gurung, F. Fabrizi, M. Ramakrishnan, L. Boie, M. J. Neugebauer, B. Burganov, M. Burian, S. L. Johnson, K. Rossnagel, and U. Staub, Correlation between electronic and structural orders in 1T-TiSe 2 , arXiv:2006.08983.

[62] W. Kuch and C. M. Schneider, Magnetic dichroism in valence band photoemission, Rep. Prog. Phys. 64, 147 (2001).

[63] F. Herman and S. Skillman, Atomic Structure Calculations (Prentice-Hall, Englewood Cliffs, NJ, 1963). 